\title{
Efeito da prática regular de atividade física no desempenho motor em idosos
}

CDD. 20.ed. 152.3

796.018
Paula Cristina dos Santos RODRIGUES* João Manuel Pardal BARREIROS ${ }^{* *}$ Maria Olga Fernandes VASCONCELOS* Suzana Raquel Moreira CARNEIRO*
*Faculdade de Desporto, Universidade do Porto - Portugal.

${ }^{* *}$ Faculdade de Motricidade Humana, Universidade Técnica de Lisboa - Portugal.

\section{Resumo}

Os efeitos do envelhecimento contribuem para uma maior lentidão no processamento da informação, nomeadamente ao nivel do tratamento da informação sensorial, decisão e programação dos movimentos. 0 presente estudo investigou o tempo de reação simples e o tempo de antecipação-coincidência, em idosos praticantes e não praticantes de atividade física. Constituiram a amostra 66 idosos de ambos os sexos com idade cronológica entre os 65 e os 89 anos $(M=74,3 ; S D=6,0$ anos), dos quais 34 eram praticantes de atividade física regular e 32 não praticantes de atividade física regular. Os instrumentos utilizados foram o "Multi-Choice Reaction Time Apparatus" para avaliar o tempo de reação simples (TRS) e o "Bassin Anticipation Timer" para avaliar a antecipação-coincidência (AC). Estas capacidades são relevantes no bemestar e funcionalidade da população mais envelhecida. Cada participante realizou com a mão preferida um total de seis tentativas na tarefa de TRS e 20 tentativas na tarefa de AC. Os resultados revelaram que o grupo de praticantes de atividade física demonstrou desempenhos superiores tanto na tarefa de TRS como na tarefa de AC. 0 sexo foi fator influente apenas no grupo de praticantes no desempenho das duas tarefas, sendo os homens mais rápidos e precisos do que as mulheres. Um processamento da informação mais rápido nos homens pode estar na base destes resultados. Como as duas capacidades avaliadas neste estudo constituem uma expressão da integridade funcional do sistema nervoso central, os resultados sugerem que a prática de atividades motoras sistemáticas pode retardar e ser um importante protetor contra os efeitos de envelhecimento nos processos perceptivo-motores inerentes a este tipo de tarefas.

Unitermos: Performance manual; Tempo de reação; Antecipação-coincidência.

\section{Introdução}

Sabemos que o envelhecimento promove um declínio gradual da capacidade funcional (LAURIENTI, Burdette, Maldjian \& Wallace, 2006), fazendo com que a informação interna e externa do organismo seja recolhida de forma menos precisa, levando muitas vezes à produção de respostas menos rápidas ou desajustadas. Consequentemente, as pessoas idosas possuem uma maior dificuldade em fazer uso da informação sensorial, em detectar e corrigir erros, e em controlar as açôes motoras quando se deparam com exigências do ambiente em que vivem (Cinelli, Patla \& STUart, 2008).

O declínio observado com o envelhecimento pode ser substancialmente modificado por influência de alguns fatores, como a atividade física
(Westlake \& Culham, 2007), o controle do peso ou o tipo de dieta (SHEPHARD, 2002). A prática de atividade física por indivíduos idosos, por exemplo, proporciona melhorias ao nível funcional (PrIOLI, Freitas Junior \& Barela, 2005), da performance cognitiva (TownSEND, ADAMO \& HaIst, 2006) e do processamento da informação (CHRISTENSEN, PAYNe, Wughalter, Yan, Henehan \& Jones, 2003).

O tempo de reação (TR) constitui uma expressão da velocidade de processamento da informação (Bashore, Ridderinkhof \& Molen, 1997) e, indiretamente, da integridade funcional do sistema nervoso central (SNC) (SHEPHARD, 2002). O declínio da velocidade de resposta em tarefas de TR ao longo do 
envelhecimento tem sido amplamente demonstrado (Der \& Deary, 2006; Hillman, Belopolsky, Snook, Kramer \& McAuley, 2004; Hultsch, MacDonald \& Dixon, 2002). Hultsch, MacDonald e Dixon (2002), por exemplo, estudaram a variabilidade da performance de jovens e de idosos no TR, provando que com a idade aumenta a diversidade entre indivíduos, aumenta a variabilidade da resposta entre diferentes tarefas, assim como a variabilidade individual da resposta na mesma tarefa. Os resultados indicaram que o nível de variabilidade foi superior nos indivíduos mais velhos e que as diferenças se acentuavam com a complexidade da tarefa. Esta lentidão progressiva é atribuída, pela maioria dos investigadores, à ineficácia dos processos de controle.

$\mathrm{O}$ desempenho em tarefas de tempo de reação também parece ser afetado pelo fator sexo. Em tarefas de tempo de reação simples (TRS) tem sido observada uma superioridade do sexo masculino relativamente ao feminino (Dane \& Erzurumluoglu, 2003; JeEves \& Moes, 1996; Lord, Castell, Corcoran, DayHew, Matters, Shan \& Williams, 2003).

A literatura que relaciona as variáveis atividade física e TR em idosos aponta para um efeito positivo do envolvimento em atividades físicas (ETNIER, Sibley, Pomeroy \& KaO, 2003; Lord et al., 2003). Estes estudos confirmam a sugestão de SPIRDUSO (1994) de que a atividade física parece não só melhorar as componentes centrais do TR, nomeadamente ao nível do SNC, como também as periféricas, estimulando a circulação sanguínea nas extremidades do corpo e propiciando temperaturas adequadas à rápida transmissão de sinais nervosos aos músculos.

A capacidade de antecipação-coincidência (AC), ao exigir que o sujeito antecipe a posição futura de um determinado objeto em movimento e que organize a sua resposta motora para que esta coincida com a chegada desse objeto ao local predeterminado, avalia a competência para predizer eventos no tempo e ajustar a resposta de sincronização. É uma competência muito solicitada em ações reais, basicamente presente em todas as ações em que a estimativa do desenrolar de eventos se deve integrar na organização da ação. Pela sua importância, a deterioração desta competência pode surgir associada a ocorrência de acidentes como quedas ao entrar em esteira rolante, atropelamentos, etc.

Ao longo do processo de envelhecimento, a investigação tem revelado uma degradação do desempenho em tarefas de AC, isto é, perda de precisão, de consistência e atraso nas respostas (MEEUWSEN, Goode \& Goggin, 1997; Pinheiro \& CorrêA, 2005), presenciando-se um declínio significativo no desempenho dos indivíduos sobretudo a partir dos 70 anos de idade (Lobjois, Benguigui \& BerTsCH, 2005; Santos, Corrêa \& Freudenheim, 2003). Em contraste com estes efeitos negativos da idade, existe alguma evidência de que os idosos permanecem em condições de realizar com precisão este tipo de tarefas em determinadas circunstâncias, tais como serem fisicamente ativos (CHRISTENSEN et al., 2003; Lobjois, Benguigui \& Bertsch, 2005). Porém, os efeitos negativos da idade em indivíduos fisicamente ativos ainda são observáveis, pois a sua performance permanece inferior à dos adultos jovens (HAYWOOD, 1980; TEIXEIRA, 2006).

$\mathrm{Na}$ população adulta, certos autores (BRADY, 1996; Williams \& JasieWICZ, 2001) têm observado uma superioridade no desempenho do sexo masculino relativamente ao feminino nas tarefas de AC, que pode estar associada a efeitos de variáveis sócio-culturais (BRADY, 1996), a solicitações mais moderadas desta competência em mulheres (BRADY, 1996; WILLIAMS \& JASIEWICZ, 2001), ou a uma participação mais elevada em desportos com exigências antecipatórias (KUHLMAN, 1992).

Tal como no TR, a AC em idosos pode ser influenciada pela atividade física, sendo evidente uma maior precisão e menor variabilidade no desempenho dos sujeitos mais ativos (CHRISTENSEN et al., 2003; DeL Rey, 1982). Christensen et al. (2003), por exemplo, estudaram indivíduos agrupados em três níveis de atividade (intenso, moderado e reduzido) quanto ao TRS, tempo de reação de escolha (TRE), tempo de AC e consumo máximo de oxigênio ( ${ }^{\mathrm{O}} \mathrm{O}_{2}$ máx). No que concerne aos valores do TRS e de AC, constatou-se que o grupo dos indivíduos muito ativos obteve performances significativamente superiores às encontradas no grupo dos indivíduos moderadamente ativos e no dos indivíduos pouco ativos. Não se encontraram diferenças significativas entre grupos nos valores do TRE. Os autores concluíram que níveis superiores de intensidade da prática de atividade física parecem estar relacionados com parâmetros fisiológicos e efeitos consequentes na performance psicomotora.

O presente estudo tem como objetivo geral investigar o TRS e o tempo de $\mathrm{AC}$ da mão preferida, em idosos praticantes e não praticantes de atividade física. De igual forma, pretendemos analisar os resultados em função do sexo. Partimos do pressuposto que os idosos praticantes de atividade física, bem como os idosos do sexo masculino, apresentam desempenhos superiores tanto no TRS como na AC em relação aos idosos não praticantes de atividade física e aos idosos do sexo feminino. 


\section{Métodos}

\section{Sujeitos}

Tomaram parte de forma voluntária neste estudo 66 indivíduos com idade cronológica entre os 65 e os $89 \operatorname{anos}(\mathrm{M}=74,3$; $\mathrm{SD}=6,0$ anos), sendo 34 praticantes de atividade física regular (20 do sexo feminino e 14 do sexo masculino) e 32 não praticantes de atividade física regular (15 do sexo feminino e 17 do sexo masculino). Os indivíduos praticantes de atividade física estavam integrados num projeto municipal de atividade física. $\mathrm{O}$ tipo de modalidade efetuada por estes idosos incluía ginástica $(\mathrm{n}=44)$, natação $(\mathrm{n}=$ $40)$, tai-chi $(\mathrm{n}=38)$, bocha $(\mathrm{n}=3)$ e caminhada ( $\mathrm{n}$ $=8$ ), podendo cada indivíduo frequentar mais do que uma modalidade. O tempo de prática deste grupo variava entre os três meses e os três anos. Os indivíduos não praticantes frequentavam centros de dia. Uma das limitaçốes deste estudo prende-se com a composição dos grupos de idade. A dificuldade de encontrar indivíduos com mais de 80 anos no projeto municipal de atividade física e indivíduos com menos de 70 anos nos centros de dia impossibilitou a análise do desempenho através da idade.

Os critérios de inclusão foram a idade (mais de 65 anos) e, no caso dos praticantes, a frequência de sessões organizadas de atividade física mínima de duas vezes por semana, ao longo de pelo menos um ano. Foram excluídos do estudo sujeitos com patologias neurológicas diagnosticadas (e.g. Alzheimer, Parkinson, Acidentes Vasculares Cerebrais), sujeitos com alterações a nível físico (especialmente a nível dos membros superiores), perceptivo ou cognitivo, indivíduos submetidos a medicação suscetível de alterar o estado de vigilância, sujeitos com visão inadequada à realização dos testes, e ainda indivíduos residentes em lares ou institucionalizados.

Os procedimentos experimentais foram aprovados pelo Comitê de Ética em Pesquisa local.

\section{Instrumentos e tarefas}

Os instrumentos utilizados nesta pesquisa foram o "Multi-Choice Reaction Time Apparatus" (modelo 63014 A) para avaliar o TRS e o "Bassin Anticipation Timer da Lafayette Instruments” (modelo no 50575), para avaliar a AC. Na tarefa do TRS pretendia-se que o indivíduo reagisse o mais rápido possível a um estímulo visual. $\mathrm{Na}$ tarefa de $\mathrm{AC}$ pretendia-se que o sujeito coincidisse a sua resposta com a chegada de um estímulo luminosos a um local predeterminado.

\section{Delineamento e procedimentos}

O objetivo deste estudo foi explicado aos participantes e foi obtido um termo de consentimento informado em que foram apresentados os procedimentos, duração do experimento e os seus direitos como participantes de pesquisa. Não foi fornecido incentivo para a participação neste estudo.

Os sujeitos foram testados individualmente, realizando em primeiro lugar a tarefa de TRS e em seguida a de AC. Foram dadas instruções verbais sobre cada aparelho e sobre cada tarefa, durante as quais os participantes observaram o seu funcionamento. $\mathrm{Na}$ tarefa de TRS os sujeitos estavam sentados em frente ao aparelho de resposta, tendo como objetivo proceder à pressão de uma chave de resposta previamente indicada pelo pesquisador. Cada participante realizou um total de seis tentativas com a mão preferida na chave do lado direito. Na tarefa de AC foi regulada a altura de sentado para homogeneização das condições de realização da tarefa. O banco estava posicionado na continuação da última calha e a 1,5 m desta, para que a propagação do estímulo visual se apresentasse no plano sagital. O objetivo da tarefa consistiu em fazer coincidir a resposta com o acendimento do último díodo da calha. Foram realizadas 20 tentativas (organizadas em quatro blocos de cinco tentativas) com a mão preferida, precedidas de duas tentativas, não analisadas, para habituação à tarefa. A velocidade do estímulo foi constante de $268 \mathrm{~cm} / \mathrm{s}$, com um intervalo inter-tentativas de $10 \mathrm{~s}$. A duração do "foreperiod" foi de 0,5 s e não foi fornecido conhecimento dos resultados em nenhuma das tarefas.

\section{Análise estatística}

A análise inferencial foi conduzida separadamente para cada tarefa, comparando-se os desempenhos dos grupos por meio de análises de variância de três fatores (sexo, grupo, tentativas), com medidas repetidas no último fator, para cada uma das variáveis. Em relação ao TRS, foi calculada a média e o desvio padrão do desempenho. No que diz respeito à $\mathrm{AC}$, foram calculados os erros absoluto (EA), constante (EC) e variável (EV) para cada participante. Em todas as análises foi adotado o nível mínimo de significância de $5 \%$. Um aspecto que poderia ser apontado como fator interveniente nos resultados é o tempo de prática no grupo de praticantes de atividade física. Assim, foi realizada uma análise preliminar, de forma a averiguar a 
influência deste fator no desempenho das duas tarefas. Os resultados não revelaram um efeito significativo deste fator em nenhuma das tarefas e, por esta razão, não foi considerado na análise a seguir discriminada.

\section{Resultados}

Os resultados serão apresentados separadamente para cada uma das tarefas.

\section{Tempo de reação}

A análise dos resultados evidenciou um efeito significativo dos fatores grupo $[\mathrm{F}(1,61)=10,667 ; \mathrm{p}=$ $0,002]$ e tentativas $[F(5,57)=3,518 ; \mathrm{p}=0,008]$. Os praticantes de atividade física foram significativamente mais rápidos $(\mathrm{M}=0,44 ; \mathrm{SD}=0,14 \mathrm{~s})$ do que os não praticantes $(M=0,58 ; S D=0,20 s)$. No contraste entre as tentativas, o teste "post hoc" (Bonferroni) demonstrou diferenças entre a sexta tentativa e a primeira, segunda e terceiras tentativas. Nenhum outro efeito ou interação foi estatisticamente significativo. Apesar dos níveis de desempenho serem claramente diferentes entre grupos, essas diferenças não foram alteradas ao longo das tentativas, o que é corroborado pela ausência de interação entre os fatores tentativas e grupo. Foi ainda investigado o efeito do sexo no TR, em cada um dos grupos. Os resultados revelaram um efeito significativo apenas no grupo de praticantes [ $\mathrm{t}(32)=2,062 ; \mathrm{p}=0,049]$. Os homens foram significativamente mais rápidos $(M=0,49$; $\mathrm{SD}=0,14 \mathrm{~s})$ do que as mulheres $(\mathrm{M}=0,39 ; \mathrm{SD}=0,14$ s). Na FIGURA 1 está ilustrado o comportamento de ambos os sexos em cada grupo ao longo das seis tentativas.

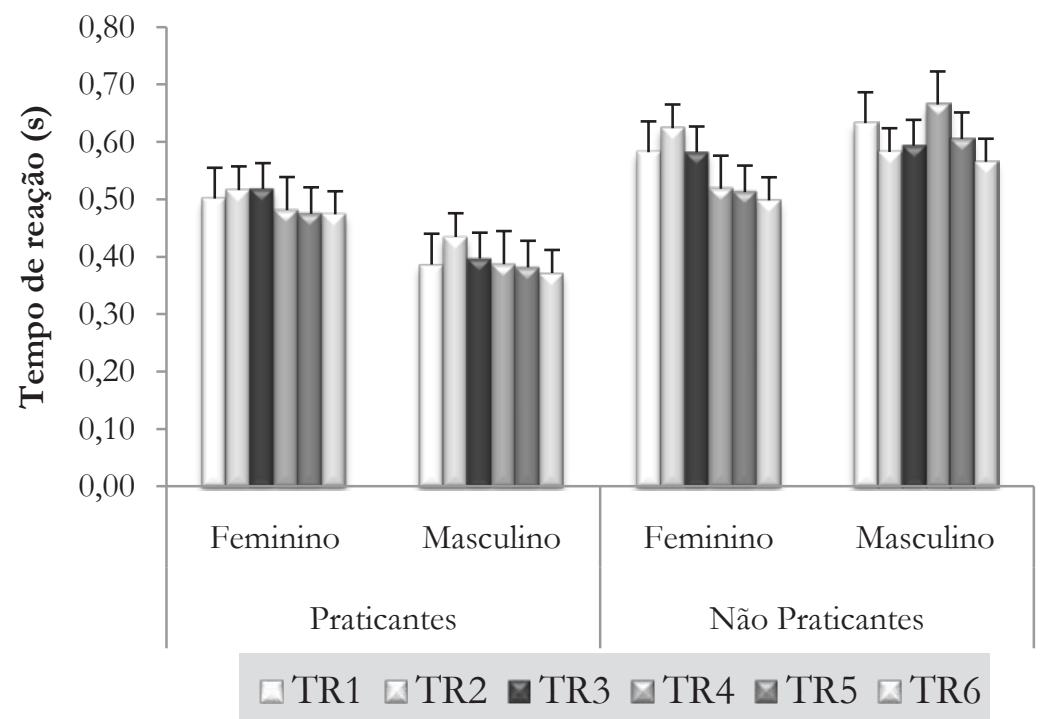

FIGURA 1 - Médias e desvios-padrão do tempo de reação (s) ao longo das seis tentativas (TR1, TR2, TR3, TR4, TR5, TR6), em função do sexo e do grupo.

\section{Antecipação - coincidência}

A precisão das respostas numa tarefa de AC é avaliada pelo intervalo de tempo entre a chegada do estímulo ou objeto a um determinado ponto e a resposta do sujeito a esse mesmo estímulo (EA); pela direção, através do atraso ou antecipação da resposta (EC), e pela consistência, traduzida pelo aumento ou diminuição da variabilidade do desempenho (EV).

Os resultados da análise destas medidas de desempenho demonstraram uma diferença significativa no EA entre os grupos $[F(1,62)=32,163 ; p<0,001]$ e entre os blocos de tentativas $[\mathrm{F}(3,60)=3,333 ; \mathrm{p}=0,025]$, mas não evidenciaram interação entre grupo e blocos. Apesar do grupo de praticantes exibir um desempenho com maior precisão $(M=0,09 ; \mathrm{SD}=0,04 \mathrm{~s})$ que o grupo de não praticantes $(M=0,25 ; S D=0,15 s)$, a magnitude $d a$ diferença foi conservada ao longo dos blocos de tentativas.

No contraste entre os blocos, o teste de Bonferroni localizou diferenças significativas $(\mathrm{p}<0,05)$ entre $\mathrm{o}$ bloco 1 e os restantes blocos, indicando uma estabilização do desempenho logo após as primeiras cinco 
tentativas. Este tipo de comportamento adaptativo é comum na aprendizagem de tarefas perceptivomotoras novas. Nenhum outro fator ou interação revelou efeitos significativos. Na FIGURA 2, está ilustrado o comportamento de ambos os grupos em termos de EA, ao longo dos blocos na tarefa de AC.

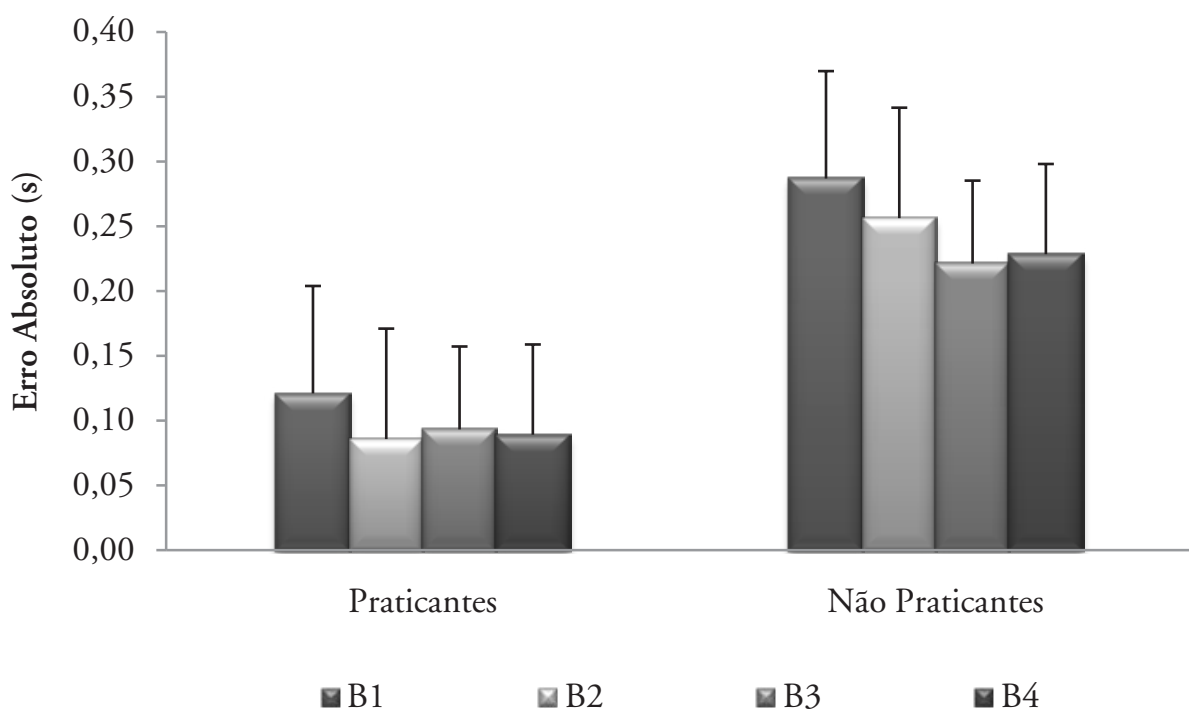

FIGURA 2 - Médias e desvios-padrão do Erro Absoluto (s) ao longo dos quatro blocos de cinco tentativas (B1, B2, B3 e B4), em função do grupo.

O perfil da curva de desempenho do EC indicia uma tendência da direção das respostas semelhante, mas nesta medida o fator grupo apresentou efeitos significativos $[\mathrm{F}(1,62)=22,771 ; \mathrm{p}<0,001]$. O grupo de praticantes obteve um erro constante significativamente inferior $(M=0,04 ; S D=0,05 s)$ ao do grupo de não praticantes $(M=0,20 ; S D=0,17$ s). Nenhum outro fator ou interação revelou efeitos significativos.
O fator grupo também evidenciou um efeito significativo quanto ao $\mathrm{EV}[\mathrm{F}(1,62)=7,416 ; \mathrm{p}=$ $0,008]$, sendo o grupo de praticantes menos variável $(\mathrm{M}=0,08 ; \mathrm{SD}=0,04 \mathrm{~s})$ do que o de não praticantes $(\mathrm{M}=0,11 ; \mathrm{SD}=0,10 \mathrm{~s})$. Nenhum outro fator ou interação revelou efeitos significativos. Na FIGURA 3, está ilustrado o perfil do desempenho de cada um dos grupos relativamente ao $\mathrm{EC}$ e ao $\mathrm{EV}$.

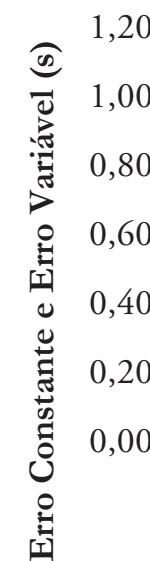

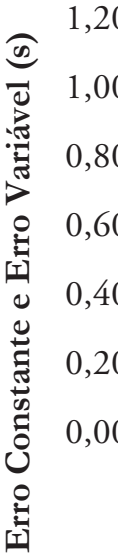

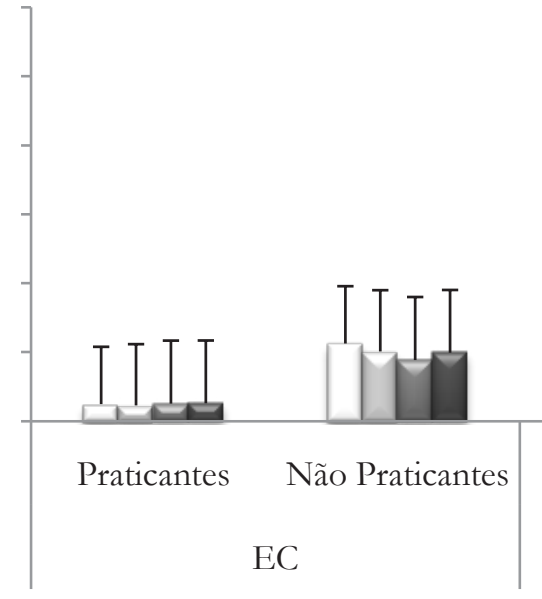

$\square \mathrm{B}$

FIGURA 3 - Médias e desvios-padrão dos Erros Constante e Variável (s) ao longo dos quatro blocos de cinco tentativas (B1, B2, B3 e B4), em função do grupo. 
$\mathrm{Na}$ tarefa de $\mathrm{AC}$, a análise do efeito do sexo em cada um dos grupos, relativamente aos EA, EC e $\mathrm{EV}$, revelou um efeito significativo apenas no grupo de praticantes, para o $\mathrm{EA}[\mathrm{F}(1,32)=10,467 ; \mathrm{p}=$ $0,003]$ e para o $\mathrm{EC}[\mathrm{F}(1,32)=5,041 ; \mathrm{p}=0,032]$. Os homens foram significativamente mais precisos (EA: $M=0,07 ; S D=0,03$ s; EC: $M=0,03 ; S D=$ 0,05 s) do que as mulheres (EA:M $=0,11 ; \mathrm{SD}=$

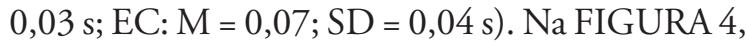
estão ilustrados os perfis do desempenho de cada um dos sexos no grupo de praticantes relativamente à capacidade de antecipação-coincidência (EA e EC).

Em síntese, os resultados deste estudo revelaram que o grupo de praticantes de atividade física demonstrou desempenhos superiores tanto na tarefa de TRS como na tarefa de AC. O sexo foi fator influente apenas no grupo de praticantes no desempenho em qualquer das tarefas, sendo os homens mais rápidos e precisos do que as mulheres.

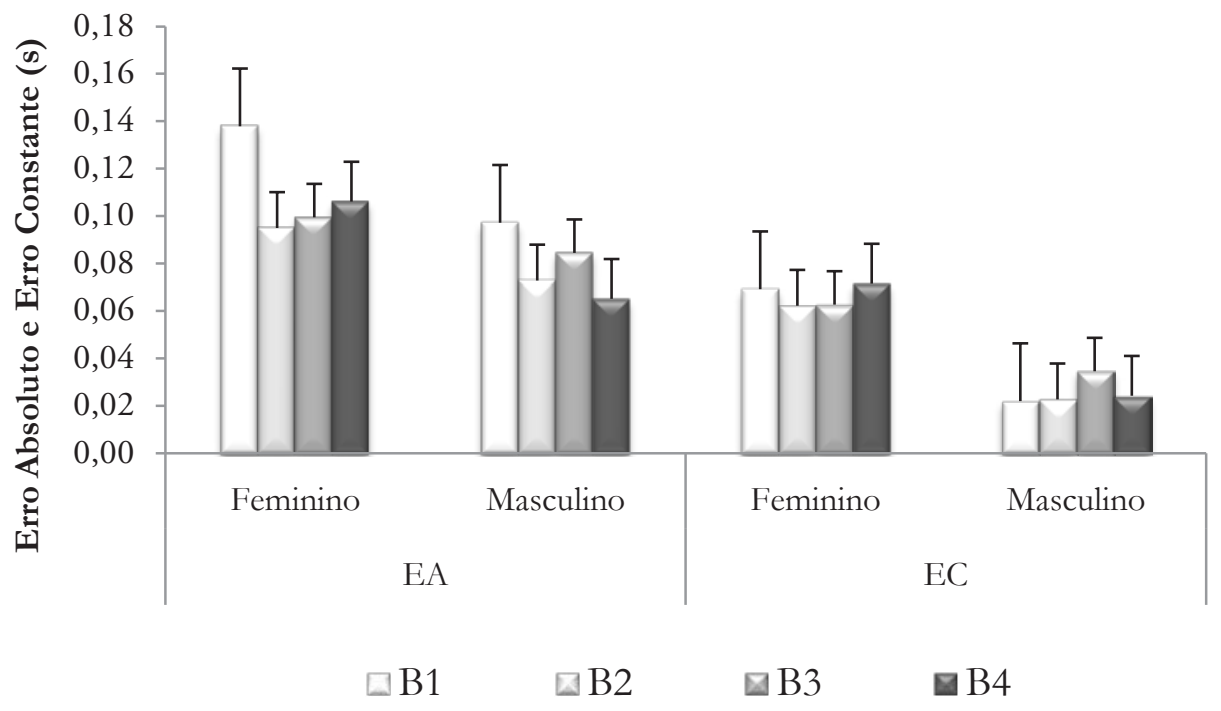

FIGURA 4 - Médias e desvios-padrão dos Erros Absoluto e Constante (s) ao longo dos quatro blocos de cinco tentativas (B1, B2, B3 e B4) no grupo de praticantes, em função do sexo.

\section{Discussão}

Este estudo pretendeu analisar o efeito da prática regular de atividades físicas nos tempos de reação simples e de antecipação-coincidência. Os resultados apontaram para performances mais elevadas do grupo de praticantes comparativamente ao grupo de não praticantes, nas duas tarefas. Este fato confirma o que tem sido já descrito na literatura, sobre o efeito da prática de atividade física em processos perceptivo-motores, a qual conduz a um desempenho mais preciso e a um uso mais efetivo da informação (Christensen et al., 2003; Del Rey, 1982; Hillman, Erickson \& Kramer, 2008; Kramer, EricKSON \& Colcombe, 2006; RaczeK \& SLEZYNSKI, 1993).

Os efeitos da atividade física sobre o TR, simples ou de escolha, estão razoavelmente identificados. Tempos de reação mais curtos em indivíduos praticantes de atividades físicas, comparativamente a não praticantes, parecem ser o resultado de um controle da função executiva mais eficaz, particularmente nos processos de atenção e memória (HILlMAN, BELOPOLSKY, SNOOK, KrAMER \& McAuley, 2004), e de uma eficiência mais elevada dos processos relacionados com a informação neurosensorial (ERICKSON, PrAKASH, Voss, CHADDOCK, Hu, Morris, White, WójCicki, McAuley \& Kramer, 2009; Gauchard, Gangloff, Jeandel \& Perrin, 2003) no que respeita às fases do processamento da informação: com a prática, os sujeitos tornam-se mais capacitados para detectar e reagir mais rápido a estímulos, selecionar as respostas em conformidade e programá-las de acordo com a seleção efetuada. Assim, a prática de uma atividade física parece gerar um aumento da velocidade do processamento 
cognitivo e, consequentemente, respostas motoras mais apropriadas.

$\mathrm{O}$ efeito da atividade física em pessoas mais velhas sobre a competência de AC é, contudo, pouco conhecido. Os nossos resultados vão ao encontro dos observados em outros estudos (CHRISTENSEN et al., 2003; Lobjois, Benguigui \& Bertsch, 2005) e mostraram, para esta variável, uma tendência semelhante à encontrada para a medida do TRS, ou seja, melhores desempenhos nos indivíduos praticantes de atividade física. Isto é particularmente importante porque este tipo de operação está envolvido com frequência em condições ou tarefas que envolvem algum risco e probabilidades elevadas de ocorrência de acidentes. Como realçam SANTOS e TANI (1995), antecipação significa preparar de antemão uma determinada situação, reduzindo, desta forma a incerteza. Manter a capacidade de antecipação durante o processo de envelhecimento poderá ser uma forma atender às exigências do ambiente, diminuindo o risco de acidentes. Quer na prática das atividades físicas quer nas atividades da vida diária, o idoso envolve-se frequentemente em ações perceptivo-motoras requerendo juízos de antecipação-coincidência. Atravessar a rua, conduzir um automóvel, alcançar e agarrar um objeto em movimento ou simplesmente apanhar um objeto que cai, implicam juízos espaço-temporais que se unem intimamente na procura da interceção de trajetórias, nos dois últimos casos, ou na tentativa de evitar essa interceção, nos dois primeiros casos. Em qualquer das situaçôes apresentadas, é solicitada a capacidade de antecipação-coincidência.

A hipótese de que os homens obteriam desempenhos mais elevados do que as mulheres foi parcialmente suportada. Efetivamente, apenas no grupo de praticantes foi evidente a superioridade do sexo masculino em relação ao feminino, corroborando os resultados alcançados por outros investigadores quer na tarefa de TRS (DANE \& Erzurumluoglu, 2003; Jeeves \& Moes, 1996), quer na tarefa de AC (BRADY, 1996; Williams \& JASIEWICZ, 2001).

Um processamento da informação mais rápido dos homens em relação às mulheres pode estar na base destes resultados no que respeita ao grupo de praticantes. Por outro lado, a inatividade pode conduzir a uma lentidão dos processos cognitivos e, desta forma, camuflar as diferenças entre os sexos. É possível que estes resultados sejam indicadores de que a inatividade propicie um declínio mais acentuado do sexo masculino, porém, torna-se necessária uma investigação mais profunda para verificar tal suposição.

Uma das limitações do presente estudo remetese ao reduzido número que conseguimos obter de praticantes com idades compreendidas entre 80-89 anos. Por esta razão, não podemos efetuar a análise da variação do desempenho em função da idade para este grupo. No entanto, efetuamos esta análise no grupo de não praticantes. Os resultados demonstraram que apesar da tendência para piores desempenhos no grupo mais velho, o fator idade não alcançou significado estatístico em ambas as tarefas. Por conseguinte, e uma vez que os estudos sobre esta temática têm utilizado tarefas simples e a análise sobre a realização da tarefa recai sempre sobre o desempenho da mão preferida, seria interessante explorar a relação entre idade, prática de atividade física e complexidade da tarefa, bem como as questóes relacionadas com a assimetria manual.

\section{Conclusão}

Os resultados do presente estudo revelaram tempos de reação mais curtos e uma maior precisão na tarefa de antecipação-coincidência no grupo de praticantes de atividades físicas comparativamente ao grupo de não praticantes. Para além disso, as diferenças entre os sexos foram apenas visíveis no grupo de praticantes. Como as duas capacidades avaliadas neste estudo revelam sinais da integridade funcional do sistema nervoso central, os resultados sugerem que a prática de atividades motoras sistemáticas pode retardar e ser um importante protetor contra os efeitos de envelhecimento no processamento da informação. Partilhamos, desta forma, com a opinião de ZEC (1995) quando refere que a melhor defesa contra os efeitos da idade na deterioração cognitiva é a prática, pois esta não permite que o desuso ocorra e, para além disso, pode criar uma capacidade de reserva maior para compensar os efeitos neurobiológicos da idade. Assim, as implicações práticas que advêm do presente estudo remetem-se à necessidade de sensibilizar os profissionais de saúde que lidam com idosos para os benefícios da prática de atividades físicas, no sentido de as implementar de forma sistemática junto deste tipo de população. 


\begin{abstract}
Effect of regular practice of physical activity in motor performance in the elderly.

The slowing down in information processing, particularly at the level of sensory information, decision and movement programming in elderly is a consequence of aging. The present study investigated simple reaction time and coincidence-anticipation in 44 physical active and in 33 non-physical active male and female elderly individuals, with chronological age between 60 - to 89-years-old ( $M=73.7 ; \mathrm{SD}=7.4$ years). The Multi-Choice Reaction Time was used to measure simple reaction time (SRT) and the Bassin Anticipation Timer was used to evaluate coincidence-anticipation (CA). These abilities play a major role in the functionality and well being of the elderly. Each participant performed with the preferred hand a total of six trials in the SRT task and twenty trials in the CA task. The results showed that the physical active group outperformed the non-physical active group in both tasks and only in this group males were better than females. A faster information process in males could underlie this outcome. Since the two abilities evaluated in this study constitute an expression of the functional integrity of the central nervous system, the results suggest that systematic practice of physical activity can delay and be an important protector against the effects of aging in the perceptual-motor processes involved in this type of tasks.
\end{abstract}

UnITERMS: Manual performance; Reaction time; Coincidence-anticipation.

\title{
Referências
}

BASHORE, T.; RIDDERINKHOF, R.; MOLEN, M. The decline of cognitive processing speed in old age. Current Directions in Psychological Science, Rochester, v.6, p.163-9, 1997.

BRADY, F. Anticipation of coincidence, gender, and sports classification. Perceptual and Motor Skills, Missoula, v.82, n.1, p.227-39, 1996.

CHRISTENSEN, C.L.; PAYNE, V.G.; WUGHALTER, E.H.; YAN, J.H.; HENEHAN, M.; JONES, R. Physical activity, physiological, and psychomotor performance: a study of variously active older adult men. Research Quarterly for Exercise and Sport, Washington, v.74, n.2, p.136-42, 2003.

CINELLI, M.; PATLA, A.; STUART, B. Age-related differences during a gaze reorientation task while standing or walking on a treadmill. Experimental Brain Research, Berlin, v.185, n.1, p.157-64, 2008.

DANE, S.; ERZURUMLUOGLU, A. Sex and handedness differences in eye-hand visual reaction times in handball players. International Journal of Neuroscience, London, v.113, n.7, p.923-9, 2003.

DEL REY, P. Effects of contextual interference on the memory of older females differing in levels of physical activity.

Perceptual and Motor Skills, Missoula, v.55, n.1, p.171-80, 1982.

DER, G.; DEARY, I.J. Age and sex differences in reaction time in adulthood: results from the United Kingdom Health and Lifestyle Survey. Psychology and Aging, Washington, v.21, n.1, p.62-73, 2006.

ETNIER, J.; SIBLEY, B.; POMEROY, J.; KAO, J. Components of response time as a function of age, physical activity, and aerobic fitness. Journal of Aging and Physical Activity, Champaign, v.11, n.3, p.319-32, 2003.

ERICKSON, K.I.; PRAKASH, R.S.; VOSS, M.W.; CHADDOCK, L.; HU, L.; MORRIS, K.S.; WHITE, S.M.; WÓJCICKI, T.R.; McAULEY, E.; KRAMER, A.F. Aerobic fitness is associated with hippocampal volume in elderly humans. Hippocampus, New York, v.19, n.10, p.1030-9, 2009.

GAUCHARD, G.C.; GANGLOFF, P.; JEANDEL, C.; PERRIN, P. P. Physical activity improves gaze and posture control in the elderly. Neuroscience Research, Shammon, v.45, n.4, p.409-17, 2003.

HAYWOOD, K.M. Coincidence-anticipation accuracy across the life span. Experimental Aging Research, Berlin, v.6, n.5, p.451-62, 1980 .

HILLMAN, C.H.; BELOPOLSKY, A.V.; SNOOK, E.M.; KRAMER, A.F.; McAULEY, E. Physical activity and executive control: implications for increased cognitive health during older adulthood. Research Quarterly for Exercise and Sport, Washington, v.75, n.2, p.176-85, 2004. 
HILLMAN, C.H.; ERICKSON, K.I.; KRAMER, A.F. Be smart, exercise your heart: exercise effects on brain and cognition. Nature Reviews Neuroscience, London, v.9, n.1, p.58-65, 2008.

HULTSCH, D.F.; MacDONALD, S.W.; DIXON, R.A. Variability in reaction time performance of younger and older adults. Journals of Gerontology: Psychological Sciences and Social Sciences, Washington, v.57, n.2, p.101-15, 2002.

JEEVES, M.A.; MOES, P. Interhemispheric transfer time differences related to aging and gender. Neuropsychologia, Elmsford, v.34, n.7, p.627-36, 1996.

KRAMER, A.F.; ERICKSON, K.I.; COLCOMBE, S.J. Exercise, cognition, and the aging brain. Journal of Applied Physiology, Washington, v.101, n.4, p.1237-42, 2006.

KUHLMAN, J.B. Coincidence anticipation: possible critical variables. Journal of Sport Behavior, Washington, v.15, p.91-105, 1992.

LAURIENTI, P.J.; BURDETTE, J.H.; MALDJIAN, J.A.; WALLACE, M.T. Enhanced multisensory integration in older adults. Neurobiology of Aging, Fayetteville, v.27, n.8, p.1155-63, 2006.

LOBJOIS, R.; BENGUIGUI, N.; BERTSCH, J. Aging and tennis playing in a coincidence-timing task with an accelerating object: the role of visuomotor delay. Research Quarterly for Exercise and Sport, Washington, v.76, n.4, p.398-406, 2005. LORD, S.R.; CASTELL, S.; CORCORAN, J.; DAYHEW, J.; MATTERS, B.; SHAN, A.; WILLIAMS, P. The effect of group exercise on physical functioning and falls in frail older people living in retirement villages: a randomized, controlled trial. Journal of the American Geriatrics Society, Baltimore, v.51, n.12, p.1685-92, 2003.

MEEUWSEN, H.J.; GOODE, S.L.; GOGGIN, N.L. Effects of aging on coincidence anticipation timing in women. Journal of Aging and Physical Activity, Champaign, v.5, p.285-97, 1997.

PINHEIRO, J.P.; CORRÊA, U.C. Desempenho em uma tarefa complexa de "timing” coincidente com desaceleração do estímulo visual em indivíduos de diferentes idades. Revista Brasileira de Educação Física e Esporte, São Paulo, v.19, p.61-70, 2005.

PRIOLI, A.C.; FREITAS JUNIOR, P.B.; BARELA, J.A. Physical activity and postural control in the elderly: coupling between visual information and body sway. Gerontology, Basel, v.51, n.3, p.145-8, 2005.

RACZEK, J.; SLEZYNSKI, J. Changes of motor efficiency in the process of human involution. In: CONFERENCE OF EGREPA: Physical Activity and Health in the Elderly, 1., 1993, Oeiras. Proceedings.... Porto: University of Porto, 1993. SANTOS, S.; CORREAA, U.C.; FREUDENHEIM, A.M. Variabilidade de performance numa tarefa de "timing" antecipatório em indivíduos de diferentes faixas etárias. Revista Paulista de Educação Física, São Paulo, v.17, p.154-62, 2003. SANTOS, S.; TANI, G. Tempo de reação e a aprendizagem de uma tarefa de timing antecipatório em idosos. Revista Paulista de Educação Física, São Paulo, v.9, p.51-62, 1995.

SHEPHARD, R. The role of physical activity in successful aging. In: CHAN, K.; CHODZKO-ZAJKO, W.; FRONTERA, W.; PARKER, A. (Eds.). Active aging. Philadelphia: International Federation of Sports Medicine, 2002. p.2-31. SPIRDUSO, W. Physical activity and aging: retrospections and visions for the future. Journal of Aging and Physical Activity, Champaign, v.2, p.233-42, 1994.

TEIXEIRA, L. Declínio de desempenho motor no envelhecimento é específico à tarefa. Revista Brasileira de Medicina do Esporte, São Paulo, v.12, n.6, p.351-5, 2006.

TOWNSEND, J.; ADAMO, M.; HAIST, F. Changing channels: an fMRI study of aging and cross-modal attention shifts. NeuroImage, Orlando, v.31, n.4, p.1682-92, 2006.

WESTLAKE, K.P.; CULHAM, E.G. Sensory-specific balance training in older adults: effect on proprioceptive reintegration and cognitive demands. Physical Therapy, Chicago, v.87, n.10, p.1274-83, 2007.

WILLIAMS, L.R.; JASIEWICZ, J.M. Knowledge of results, movement type, and sex in coincidence timing. Perceptual and Motor Skills, Missoula, v.92, n.3, pt.2, p.1057-68, 2001.

ZEC, R.F. The neuropsychology of aging. Experimental Gerontology, v.30, n.3-4, p.431-42, 1995.

\author{
ENDEREÇO \\ Paula Cristina dos Santos Rodrigues \\ R. Dr. José Marinho, 267 \\ 4460-752 - Matosinhos - PORTUGAL \\ Recebido para publicação: 15/01/2009 \\ Revisado em: 06/01/2010 \\ e-mail: packn2@gmail.pt \\ Aceito: 06/07/2010
}

